\title{
URINARY KIM-1 AS A BIOMARKER OF AMINOGLYCOSIDE-INDUCED NEPHROTOXICITY IN PREMATURE NEONATES
}

\author{
S.J. McWilliam ${ }^{1}$, D.J. Antoine ${ }^{2}$, M. Turner ${ }^{3}$, V. Sabbisetti ${ }^{4}$, J.V. Bonventre ${ }^{4}$, B.K. Park ${ }^{2}$, R.L. Smyth ${ }^{1}$, M. \\ Pirmohamed $^{2}$ \\ ${ }^{1}$ University of Liverpool, Alder Hey Children's Hospital, ${ }^{2}$ MRC Centre for Drug Safety Science, The \\ University of Liverpool, ${ }^{3}$ University of Liverpool, Liverpool Women's Hospital, Liverpool, UK, ${ }^{4}$ Bonventre \\ Lab, Brigham and Women's Hospital, Boston, MA, USA
}

Background and aims: Premature neonates are frequently exposed to aminoglycoside (AG) antibiotics, which are potentially nephrotoxic. Novel urinary biomarkers may provide a non-invasive means of identifying early changes of AG-related renal proximal tubule toxicity, to enable adjustment of treatment, and identification of infants at risk of long-term renal impairment.

Methods: Urine samples were collected from 41 premature neonates $(<32$ weeks gestation) at least once per week, and daily during courses of gentamicin and for 3 days afterwards. We assessed the associations between three urinary biomarkers (Kidney Injury Molecue-1 (KIM-1), Neutrophil Gelatinase-associated Lipocalin (NGAL), and N-acetyl- $\beta$-D-glucosaminidase (NAG)) and AG exposure.

Results: Treatment with gentamicin was associated with significant increases in KIM-1 (mean difference from not treated, $1.64 \mathrm{ng} / \mathrm{mg}$ urinary Creatinine $(\mathrm{uCr}) ; 95 \%$ Confidence Interval $(\mathrm{CI}) ; 0.54,2.75)$, NGAL (453.6ng/mg uCr; 95\%CI 145.1, 762.2), and NAG $(0.08 \mathrm{IU} / \mathrm{mg} \mathrm{uCr} ; 95 \% \mathrm{CI} 0.02,0.15)$. There was a reduction in serum creatinine $(-4.64 \mathrm{micromol} / 1 ; 95 \% \mathrm{CI}-8.64,-0.64)$ during AG treatment. When adjusted for potential confounders the treatment effect of gentamicin remained significant only for KIM-1 $(1.35 \mathrm{ng} / \mathrm{mg}$ $\mathrm{uCr} ; 95 \%$ CI $0.05,2.65$ ). Between courses of gentamicin each biomarker returned to values observed when not receiving gentamicin. Gestational age had no significant effect upon the elevation in urinary biomarkers associated with AG treatment.

Conclusions: These findings suggest that KIM-1 may have clinical utility as a sensitive and specific biomarker of AG-associated nephrotoxicity in premature neonates. 